\title{
FACEBOOK ADVERTISEMENTS FOR SURVEY PARTICIPANT RECRUITMENT: CONSIDERATIONS FROM A MULTI-NATIONAL STUDY
}

\author{
Robert Thomson \\ Hokkaido University \\ 5 Chome Kita 8 Jonishi, Kita Ward, Sapporo, Japan \\ rob.thomson@lynx.let.hokudai.ac.jp \\ Naoya Ito \\ Hokkaido University \\ 5 Chome Kita 8 Jonishi, Kita Ward, Sapporo, Japan \\ naoya@imc.hokudai.ac.jp
}

\begin{abstract}
Facebook's global reach suggests good potential for recruiting research participants and collecting objective behavioral data for cross-cultural research. Previous literature suggests the usefulness of Facebook advertisements to recruit participants in single-country studies. However, Facebook advert use in multi-country studies has not yet been reported. Nor are there any reports about soliciting Facebook user data (via Facebook applications) using Facebook advertisements. This paper contributes to this gap in Internet research, reporting on the effectiveness of Facebook advertisements to recruit participants, and for soliciting anonymized Facebook user data, in a 20-country study about privacy concerns on Facebook. Over seven days, 399 Facebook users from 18 countries responded to country-targeted advertisements in 13 languages. Response rates (ratio of advert clicks to valid responses) per country varied from $0 \%$ up to $14 \%$. Overall, two-thirds of the country response rates were below 5\%, and many country samples were gender-biased due to confounding societal factors. We conclude that for multi-national studies, Facebook advertisements may have potential for simple participant recruitment for surveys, but have limitations for soliciting Facebook user data. For user data collection, methods such as Amazon Mechanical Turk and snowball sampling may be more effective, but can be limited in their international reach.
\end{abstract}

Keywords: Internet Research Methods, Facebook Advertisements, Recruitment, Surveys, Data Collection, SNS, Cross-Cultural 


\section{INTRODUCTION}

In early 2012, we set about surveying Facebook users from around the world about their concerns regarding privacy on Facebook (the degree to which users are concerned about unwanted third party accesses to their personal information on Facebook). The task was formidable in the sense that we planned to conduct analyses on the data using multilevel modeling techniques; in order to assure statistical power in such techniques, an absolute bare minimum of 20 countries and 30 participants per country, preferably up to 100 participants per country, are required ${ }^{1}$. For a relatively small graduate school in Japan, and lacking a suitably diverse network of possible collaborators, it would be no small feat to achieve these numbers.

Our motivation for the study was to address limitations in current literature regarding cross-cultural differences in privacy concern on Facebook. That is to say, there is no doubt that societies can differ in levels of privacy concern on Facebook ${ }^{2,3,4,5,6}$. However, while many studies invoke cross-cultural paradigms such as Hofstede's ${ }^{7}$ cultural dimensions as predictors, studies tend to focus only on pairs of countries; this leads to tautological and often contradictory hypotheses which become easy to prove given the right pair of countries (contrast Krasnova \& Veltri ${ }^{4}$ and Lowry, Cao, \& Everard ${ }^{5}$ for example). Suffice it to say, we considered it necessary to sample a large number of societies, so that we might better grasp the general patterns of privacy concern variance across societies, and better apply general theories of culture as possible quantitative predictors ${ }^{8,9}$.

A large challenge facing us was gathering data, which naturally begins with recruitment of study participants. It was here, encouraged by the relative success of previous studies (see Literature Review below), we decided to attempt recruitment of participants via Facebook advertisements.

Below, we survey the current published literature chronicling the use of Facebook for participant recruitment before reporting on our own experiences in utilizing Facebook advertisements for recruiting participants for a multi-national study. To our knowledge, ours is the first paper to report the efficacy of Facebook advertisements for recruiting participants for a multi-national cross-cultural study. We also compare the efficacy of Facebook advertisement-based recruitment to other online methods, namely snowball sampling and Amazon Mechanical Turk (AMT) crowd sourcing.

\section{LITERATURE REVIEW}

As others have argued at length, utilizing Facebook as a tool for research has considerable appeal ${ }^{10}$. First and foremost, Facebook's sheer global ubiquity is phenomenal. Facebook has over 1 billion registered 
users $^{11}$, more than $80 \%$ of which hail from countries other than the United States ${ }^{10}$. Furthermore, while independent reports suggest Facebook use may be declining in the West, growth in other markets such as South America and Asia is still strong ${ }^{12}$.

Equally attractive is potential access to a depth of cross-cultural behavioral data which can, in theory, be captured independent of culturally biased self-reporting. As Kitayama ${ }^{13}$ points out, people "do not notice distinct patterns of behaviors they engage in" meaning that "retrospective verbal reporting may be suspect as evidence of what really goes on spontaneously... in the mind of people" (p. 90). It is here that data gleaned from Facebook users' Facebook accounts can be of great value. Researchers can request users to add a Facebook application to their Facebook account, which, upon user approval, will collect the individual's Facebook account data. By doing so, researchers are able to record a variety of anonymous behavioral data points (e.g., network characteristics, communication patterns, linguistic data, etc.) for ecologically compelling examinations of trends both within and across cultures ${ }^{10}$. For our purposes (studying privacy concern on Facebook), this was appealing; rather than simply asking users from different countries how concerned they are about privacy on Facebook, we could record privacy protection behaviors (such as privacy setting data) to include in our analyses as objective behavioral measures.

Difficulties arise, however, in how to actually access users' behavioral data held on Facebook servers. While data crawling can be effective for collecting user data online in some contexts, including the crawling of publicly available user information on Facebook ${ }^{14}$, increasingly stringent privacy policy terms - which unequivocally preclude collection of users' publicly available data via robots and data crawling software ${ }^{15}$ - and privacy systems mean this is an increasingly difficult task.

One relatively straightforward method of accessing users' data is to simply ask them to provide it. As mentioned previously, Facebook users can be asked to add an application to their Facebook account, and upon informed consent on the part of the user, the application can record the data the participant has allowed to be recorded. Creating or editing existing Facebook applications does require a relatively robust knowledge of coding, however Facebook has a depth of documentation available for developers (see https://developers.facebook.com).

Some researchers have successfully recruited millions of users to their applications, allowing fascinating insight into user demographics and behavior, arguably with immense cross-cultural research potential ${ }^{16}$. Some examples include "Hugged," an application launched in February 2008 allowing Facebook users to send virtual 'hugs' to their friends, and as of 
March 2010 had 28 million users, providing scores of network analysis data $^{17,18}$. The "MyPersonality" project allows Facebook users to take a variety of personality-related tests and receive feedback on their scores, and as of April 2013 the project had over 6 million test results and a record of over 4 million Facebook profiles ${ }^{19}$. This allows associations to be made, for example, between personality variables and individuals' interpersonal network size and reach ${ }^{20}$. The beauty of many of these researcher-initiated Facebook applications is that users are drawn to the applications for recreation (as in the case of Hugged) or personal enlightenment purposes (as is the case for MyPersonality); compensation to participants comes in the form of intrinsic rewards.

For many researchers however, developing a stable, bug-free, and intricate application may be beyond reach due to time constraints or lack of technical skill. Alternatively, complex applications may simply be unnecessary for one's purposes. In our case, we simply wanted to record privacy setting data, which did not require a complex application. To this end, many researchers turn to Facebook in order to simply recruit participants to online surveys, which are administered on different online survey platforms such as Survey Monkey, Limesurvey and others. As part of the survey, if participants are requested to return to Facebook to add a simple Facebook application (developed by the researchers on the Facebook platform) to their Facebook account, researchers should, in theory, be able to relatively simply gather objective behavioral data points beyond simple self-reports.

To advertise one's survey, some researchers decide to pay for advertising on Facebook, soliciting participation from Facebook users in an online survey by way of incentives. Most literature chronicling the process of recruiting survey participants via Facebook advertisements covers recruitment of hard to reach populations, such as middle and high school students (due to prohibitively restrictive parental consent requirements) ${ }^{21}$, substance use populations ${ }^{22}$, and medical trial re-recruitment ${ }^{23}$. A summary of a sample of such literature is presented in Table 1. From these studies, it appears that survey recruiters targeting Facebook users in the US, Canada and Australia for online survey participation can typically expect response rates (valid responses per advertisement clicks) of anywhere between $10 \%$ to near $40 \%$. Of the two studies reporting $3.5 \%$ and $0 \%$ response rates, one was recruiting for on-site study participation, and the other was recruiting in a very narrow age range.

Despite a thorough search, however, and to our knowledge, there are no papers which detail using Facebook advertisements for recruiting survey participants in a diverse sample of countries, in particular outside of English 
speaking countries. In addition, none of the studies outlined in Table 1 attempted to gather user data via Facebook applications.

\section{RESEARCH QUESTIONS}

When administering our study, we had two main questions that are currently unanswered by existing literature. Namely:

RQ1 : Can a researcher expect a response rate using Facebook advertisements of at least $10 \%$ in countries outside of the US, Canada, or Australia?

RQ2 : Will Facebook users be willing to take part in an online survey if participation requires that they add a Facebook application to their Facebook account?

Below, we outline our recruitment methodology before moving on to analyzing the performance of our recruitment campaign.

\section{METHODOLOGY}

\subsection{Participants}

The target population was Facebook users living in one of 18 countries listed in Table 2 in the results section.

\subsection{Facebook Recruitment Campaign}

Facebook advertisements were created using the standard Facebook advertisement system (https://www.facebook.com/advertising). As of May 2013, advertisement text is limited to 25 characters for the ad headline, and 90 characters for the ad body text. Advertisement text language was adjusted depending on the target country. See Table 2 for country-specific languages used in the advertisements. An image can be included in the advertisement. Examples of ads used in our study can be seen below in Figure 1. The same image was used in all ads. 


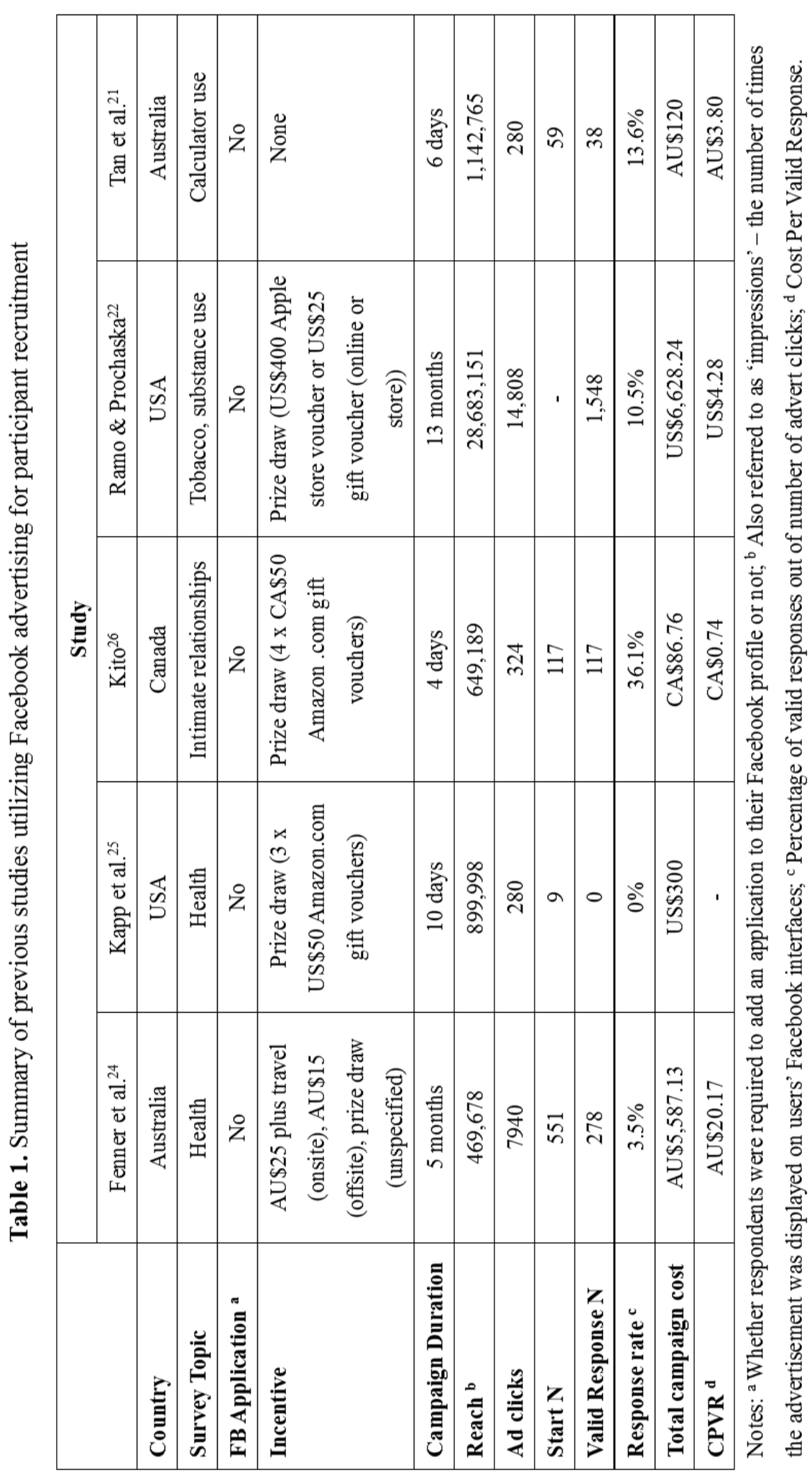


English

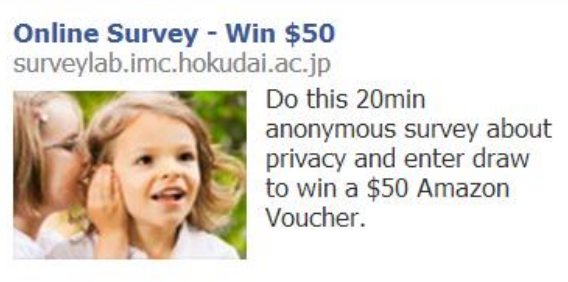

French

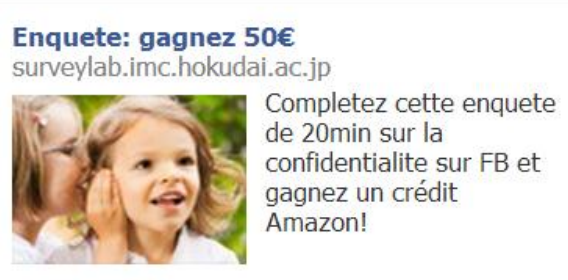

Japanese

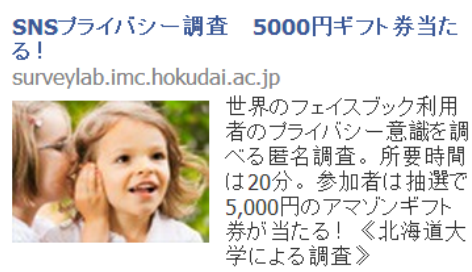

Arabic

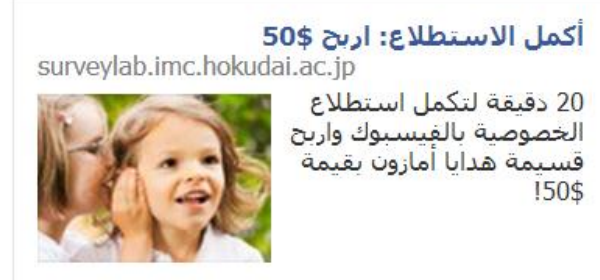

Figure 1. Example Facebook advertisement used in the study

Advertisers on Facebook have the option of paying for ads on a Cost-per-Click (CPC) basis or per 1,000 impressions. We opted to pay CPC, where we would enter a bid (the most one is willing to pay per click) on a per-click basis in each country (suggested bid amounts vary country to country). Whether or not an ad is displayed is a product of various factors, including feedback about the ad from users and past performance (number of clicks) as well as the bid made by the advertiser ${ }^{27}$. Assuming a response rate (ratio of valid survey responses to number of ad clicks) of $30 \%$ in each of the 18 countries, and a desired number of valid responses of 75 responses per country, we calculated how many clicks we would need per country (in our case, 250 clicks per ad per country). From this total required number of clicks per country, a total campaign budget per country based on Facebook's suggested CPC bid amount, which varied from US $\$ 0.12$ (Tunisia) to US $\$ 1.53$ (Japan), was calculated.

For example, in order to gather 75 valid responses from Poland, and assuming a 30\% response rate, we would need ads displayed to Facebook users in Poland to be clicked 250 times. Multiplying the required number of clicks by the suggested click bid price for Facebook ads in Poland at the time (US\$0.23 per click) gave a campaign budget of US\$57.48 for the Polish ads. We then set each country's ad campaign to run for 7 days $\left(23^{\text {rd }}\right.$ to $30^{\text {th }}$ July, 2012). With the above information, Facebook's algorithm then evenly splits each country's budget up into equal daily amounts, and 
displays the ads each day to users until the daily click budget is met. In the case that the full daily budget is not used on any particular day (did not receive enough clicks), the remaining balance is carried over to the next day in the campaign.

Clicking on a language-specific survey link directed participants to a page outlining the purpose of the survey (the privacy concern survey described in the Introduction), a statement that the survey would take approximately 15 minutes to complete, assurances of anonymity, a privacy policy link, and an explanation that as part of the survey, participants would be required to add a Facebook application which would record only their privacy settings for their 50 most-recent Facebook status updates, photos, photo albums, videos, and notes. Also included was a statement that under no circumstances would any identifying information be collected via the Facebook application. An explanation of how to remove the application after participation was also included.

As an incentive for participation, participants recruited via Facebook ads or the snowball sampling method could provide an email address to enter into a random draw for one of two US\$50 online Amazon Gift Voucher (this draw was not offered to the AMT participants, who were compensated US\$1 for their time). Where online Amazon Gift Vouchers were available in local currency, a gift voucher draw equivalent to US\$50 in the local currency was offered.

\subsection{Snowball Recruiting}

We also attempted to recruit participants (to the same privacy concern survey) via snowball sampling during the Facebook ad campaign period, to make up for a poor response from the Facebook ads (see Results section below). Snowball sampling was conducted in two ways: 1) requests to share the survey link on Facebook were sent out to the lead researcher's 1,400 Facebook contacts, both as public requests for shares, as well as numerous private messages; and 2) messaging the administrators of publically searchable Facebook groups likely to be able and willing to assist in sharing the online survey link (such as the "Tunisia \& Japan forever ever and ever" group on Facebook (https://www.facebook.com/tunijapan)).

\subsection{Amazon Mechanical Turk}

To further bolster participant numbers for the privacy concern survey outlined in the introduction, we also utilized Amazon Mechanical Turk (AMT), attempting to make use of the 'international worker pool' available. AMT is a web-based crowd-sourcing platform whereby 'requesters' can offer monetary rewards to 'workers' for simple tasks such as completing online surveys. Previous research suggests surveys completed by workers 
on AMT provide reliable data, and respondents from the US are generally representative of the broader Internet-using population ${ }^{28,}{ }^{29}$. The same studies also paint AMT as useful for accessing a global pool of workers. We posted survey tasks on AMT in several languages, offering US $\$ 1$ per completed survey (workers were prevented from filling the survey out more than once).

\subsection{Study Procedures}

The Hokkaido University review board approved the study procedures, as outlined below. We prepared an online survey administered via Limesurvey installed on our own university server with an .ac.jp domain name, the standard academic top-level domain extension in Japan. The survey included demographics and measures regarding privacy concern, as well as socio-ecological variables, and some personality measures. Participants were told the survey would take 15 minutes, however in reality it generally took 8 to 10 minutes. The survey was prepared in 13 languages, all translated by professional translators from English to the target language (see Table 2 for a list of languages). All translations were independently checked for errors by a second professional translator and edited where necessary.

\section{RESULTS}

A summary of the Facebook advertisement performance in each country, as well as recruitment numbers via the snowball and AMT recruitment methods can be found in Table 2. In Pakistan and the Philippines, AMT was the only recruitment method used. In total, we spent US $\$ 2,099.07$ on the seven day Facebook ad campaign, with individual country campaign costs ranging from US\$28.31 in Tunisia to US\$329.11 in Japan. Note that performance in terms of attracting clicks was reasonably acceptable in most countries; ads in 13 countries attracted more than 250 clicks, with the remaining 5 countries' ads receiving over 200 clicks each. From this, one can conclude that the ads were successful in attracting potential participants to the survey landing page.

Actual response rates, however, did not fare nearly as well; in all but two countries, the response rate was below $10 \%$, with 12 countries' response rates at below 5\%. While the overall average cost per valid response is a respectable US $\$ 5.26$, in countries such as Germany, The Netherlands, USA, Brazil, Poland and France, low response rates in these countries meant an exorbitant cost per valid response (see highlighted cells in Table 2). Plenty of Facebook users clicked on the advertisements, but did not proceed past the survey landing page. 


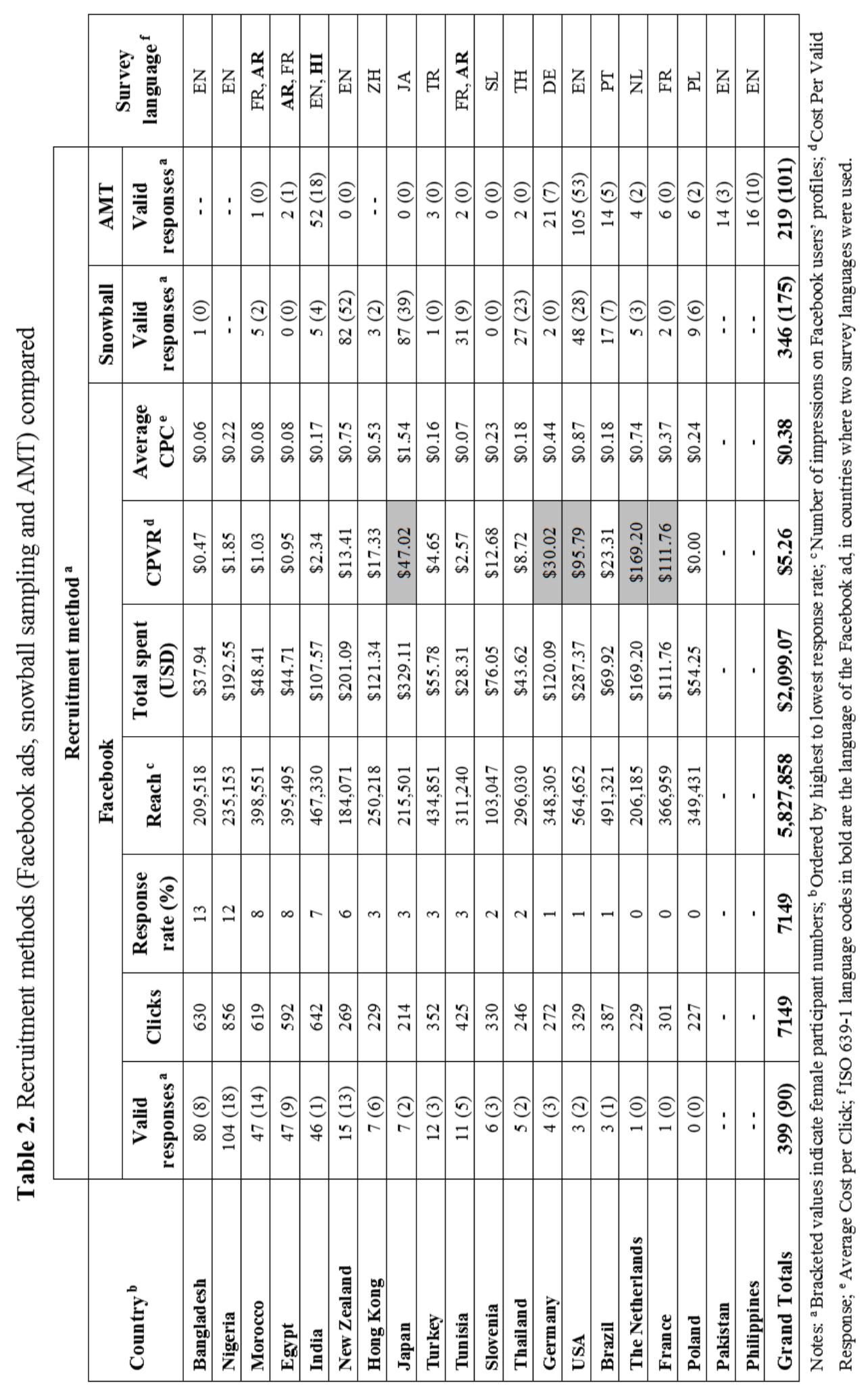


Also note the large gender bias in the Facebook ad sample, as $75 \%$ of all participants who provided valid responses after being solicited via Facebook ads are male. Indeed, the percentages of female participants in samples from India (2\%), Bangladesh (10\%), Nigeria (17\%), and Egypt (20\%) are unacceptably low. Sex ratios in total valid response numbers garnered via snowball sampling and AMT (approximately 51\% and $41 \%$ female, respectively) are more balanced.

\section{DISCUSSION AND POST-HOC ANALYSIS}

It is difficult to consider our efforts to recruit survey participants and solicit user data solely via Facebook advertisements a success in this case. As for why our response rates were so low in the Facebook advertisements, we suspect this has much to do with the requirement for participants to add a Facebook application. Note that in previous online-survey recruitment in the US and Canada garnered response rates of up to $30 \%$. Our response rate for the US was less than $1 \%$. Perhaps survey participants residing in the US, for example, feel reluctant to add an application due to distrust in unknown Facebook applications. In retrospect, we also acknowledge that such distrust may have been made stronger by the very subject of our survey: privacy. With so much talk of protecting participants' privacy on the survey landing page, potential participants may have been primed to be wary, thus increasing feelings of distrust.

Interestingly, however, despite possible trust concerns, there were countries where response rates were better; $13 \%$ in Bangladesh, near 12\% in Nigeria (see Figure 2). We noted that those countries where response rates were relatively high also tended to have relatively low GDP. Indeed, this observation held up against statistical analysis; a linear regression analysis with the US\$50 reward value (converted to an 'objective' value defined as percent of GDP per capita) as independent variable and response rate as dependent variable showed a strong and significant relationship $(b=.89, p$ $\left.<.001, R^{2}=.80\right)$. In equation form, with unstandardized coefficients,

$\mathrm{Y}_{\text {RESPONSE RATE }}=.009+5.39 \mathrm{X}_{\text {REWARD VALUE (percent of GDP PER CAPITA) }}$

where for every 1 unit increase in reward value (as percent of GDP per capita) equates to a $5.39 \%$ increase in response rate. In other words, our offered reward of a US\$50 Amazon.com gift voucher draw equaled $0.025 \%$ of GDP per capita in Bangladesh, resulting in a 13\% response rate. Assuming the relationship between response rate and objective reward value is indeed linear, then in order to attain a $13 \%$ response rate in the US we would need to have offered a US\$385-value draw. Of course, there may in 
practice be diminishing returns; intuitively we are inclined to believe US participants may be swayed with a smaller reward offer.

Naturally, the discrepancy here between the AMT and snowball methods versus the Facebook advertisements method might call this assertion (that users are unwilling to add a Facebook application) into doubt; despite reluctance for US-, Japan-, and New Zealand-based participants to take part in the survey via Facebook ads, they seemed willing to take part when recruited via other means. However, one cannot underestimate the potential risk-mitigating effects of either past experience or the recommendation of a trusted other. That is, to the general Facebook user, our survey was a completely unknown entity. There was no reason to believe that our application would not actually record much more than it purported to.

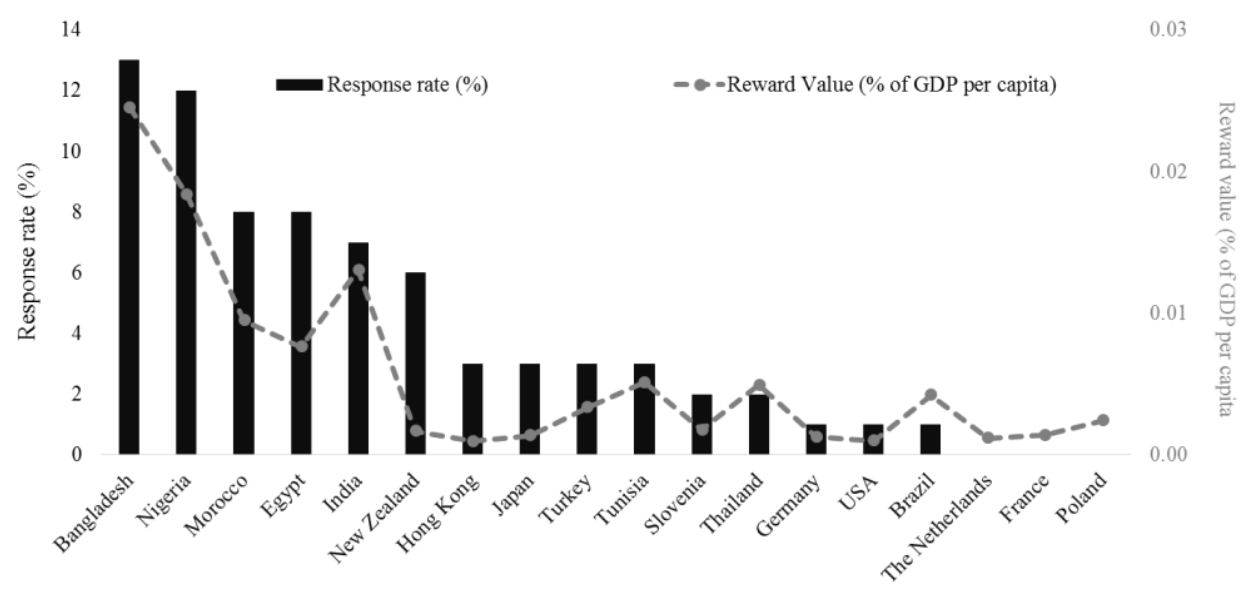

Figure 2. Response rate (\%) and objective reward value (as percent of GDP per capita) per country

For the AMT workers, however, it is fair to assume they are accustomed to participating in surveys, even perhaps adding Facebook applications for research purposes. Furthermore, one can only assume that their continued use of AMT in the first place is due to positive (or at least non-negative) experiences of AMT being the norm. They would have little reason to believe that our survey would be any different to any other survey they have taken on the platform. Reward for AMT workers is also guaranteed; they know they will receive payment for participation, which is not guaranteed for the other participants. Outside of the US and India, however, it was difficult to recruit participants via AMT, which calls into question its true usefulness in attaining a representative sample of participants outside the US and India. 
Snowball sampling seemed fairly effective; despite not having guarantee of reward, users seemed willing to participate. This is hardly surprising; the concept behind snowball sampling is that individuals come in contact with the survey via known others. If a close friend or acquaintance recommends a survey, this should carry with it a certain level of trust. Note however that the effectiveness of snowball sampling was limited to countries where the lead researcher has significant interpersonal networks: New Zealand, Japan, and the USA.

\subsection{Gender Bias and Societal Factors}

Finally, before moving on, it is important to examine in more depth the gender discrepancy in the Facebook ads sample. As Figure 3 shows, more than 8 countries in the Facebook ads sample had less than $40 \%$ females in the sample. Hardly surprising is these countries also have a smaller percentage of females using Facebook in the first place $(r=.82, p<.001)$. This begs the question, however, why are there so few female Facebook users in some countries? This is an important methodological question worth deeper analysis, as it may point to intrinsic limitations for Facebook ad recruitment in terms of attaining a gender-balanced sample.

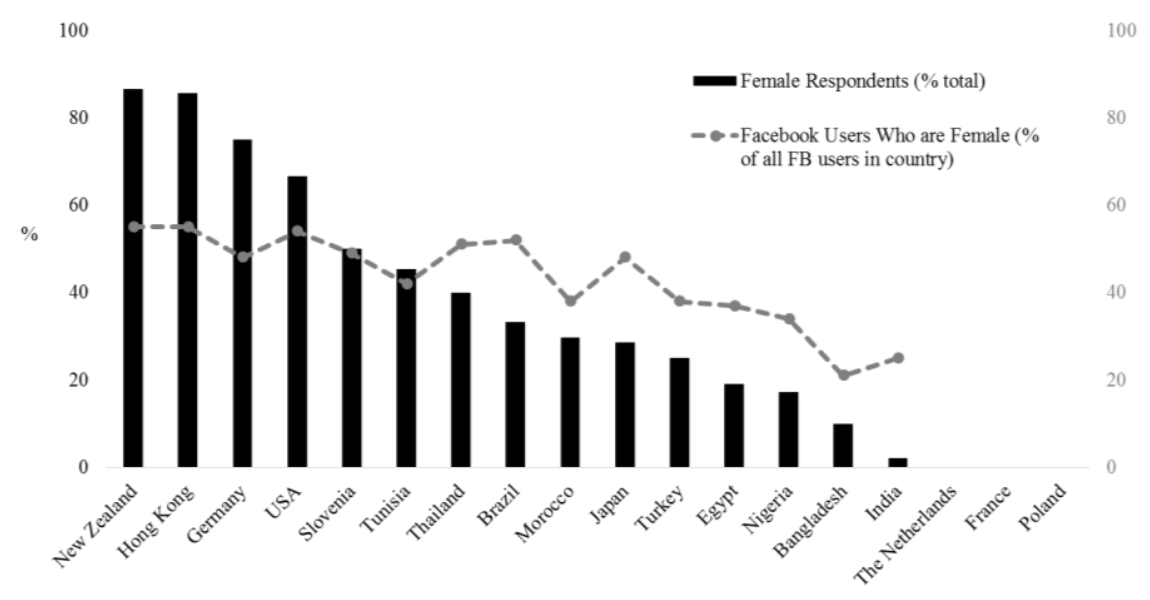

Figure 3. Female respondents (\% within-country) and Facebook users who are female (\% within-country)

A hint to understanding this phenomenon lies in the countries in our sample that showed particularly low female Facebook users: Morocco, Turkey, Egypt, Nigeria, Bangladesh, and India. It is here that Batool \& Mahmood $^{30}$ and Shafique \& Mahmood's ${ }^{31}$ research into internet café use in developing nations provides insight into this phenomenon. That is, Batool \& Mahmood $^{30}$ find a major motivation for using Internet cafes in the 
developing countries they surveyed was a lack of affordable high-speed Internet at home. Shafique $\&$ Mahmood $^{31}$ further note the relative absence of women in internet cafes, which they proffer "could well be a reflection of the secluded lives of most Pakistani women" (p. 353).

We wondered whether factors such as these might predict female Facebook user numbers across our sample of 18 countries. That is, in countries where household internet connection rates are low, and cultural values inhibiting women from frequenting internet cafes are prevalent, are there fewer women using Facebook?

To explore this question, we conducted a post-hoc moderation analysis, with country-level Internet household access (households with internet connection per 100 households $^{32}$ ) as an independent variable, female Facebook user percentage in each country ${ }^{33}$ as a dependent variable, and gender equality as a moderating variable. As a proxy for gender equality, we used country-level responses to the question "A university education is more important for a boy than for a girl" (1 - Strongly agree, 5 - Strongly disagree) from the World Values Survey ${ }^{34}$; lower values on this item indicate lower gender equality.

To put our hypothesis simply, in order for a country to be low on female Facebook users, women must have 1) no access to the Internet at home and 2) they must not be able to access Internet outside of the home; we predict norms and values against gender equality to facilitate this. That is, as gender equality drops, values inhibiting women's use of Internet cafes (i.e., congregating in public places such as cafes) should be more prevalent.

We first conducted a simultaneous regression model consisting of the two predictors (household Internet connection rate and gender equality) and an interaction term (household Internet connection rate by gender equality). Results indicated that greater gender equality $(\beta=.49, S E=.224, p<.05)$ was associated with higher female Facebook user ratio, but household Internet connection rate $(\beta=.30, S E=.223, p>.05)$ was not. The interaction term, however, was significant $(\beta=-.42, S E=-.167, p<.05)$, suggesting the effect of household Internet connection rate on female Facebook user ratio depends on societal levels of gender equality. Simple slopes for the association between household Internet connection rate and female Facebook user ration were tested for low (- 1SD below the mean), moderate (mean), and high (+1 SD above the mean) levels of gender equality. The only significant slope was that for low levels of gender equality $(\beta=.72, S E=.229, p<.01)$ (see Figure 4$)$. In other words, these results suggest in countries where gender equality is low, if household Internet connection rates are also low, women have little opportunity to access the Internet, let alone create Facebook accounts. Note that we also 
ran analyses using the UNDP Gender Inequality Index (GII) ${ }^{35}$, which revealed a similar interaction.

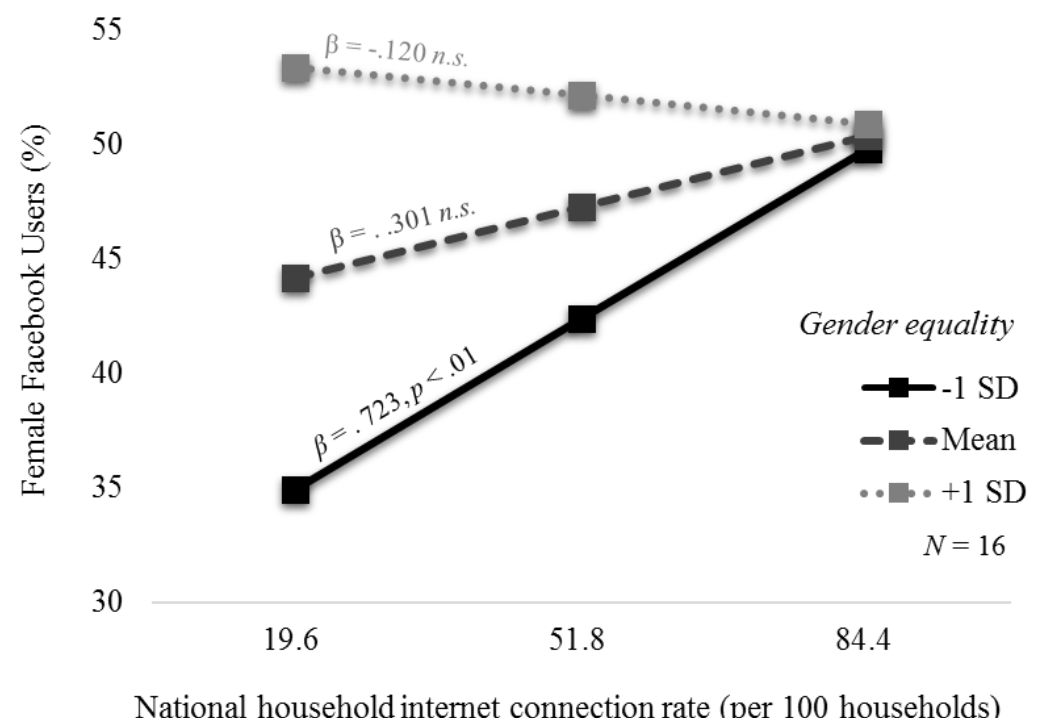

Figure 4. Moderation of the effect of national household Internet connection rate on ratio of female Facebook users by gender equality

These results suggest limitations for attaining gender-balanced samples using Facebook ads for recruitment in some societies where social factors preclude many women from accessing the Internet. Using the advertisement targeting feature when creating ads on Facebook, one could, theoretically create separate gender-specific campaigns for countries where gender imbalances are to be expected. However, if female Facebook users make up less than $30 \%$ of the user base in a country, such is the case in Bangladesh, even if one managed to attain a balanced sample, questions of representativeness arise: how representative of the larger population of women in Bangladesh are those women who use Facebook in Bangladesh?

Ultimately, our experiences leave us with room for continued experimentation with Facebook advertisements. Using Facebook advertisements, we reached almost 6 million Facebook users in 18 countries, resulting in over 200 advert clicks per country. Herein lies Facebook advertising's positive potential: If one is able to successfully convert clicks into participation, cross-cultural research has found a great ally. The negatives however are just as real; soliciting user data is problematic, gender balance is skewed, and traditional alternatives carry the same limitations as they always have - snowball sampling (despite its effectiveness) requires a 
vast personal research network, and AMT's global reach is limited (despite intimations to the contrary).

\section{CONCLUSION}

In our study of Facebook privacy concern in 20 countries, we utilized Facebook advertisements to target Facebook users in 18 countries in an attempt to recruit survey participants and solicit Facebook account privacy setting data by way of a simple Facebook application. While other studies have reported favorable response rates when recruiting via Facebook advertisements, we experienced generally unfavorable response rates for many countries in our sample. We suggest this is due to our requirement for participants to add a Facebook application which would record privacy-setting data; not many users were willing to do this after being solicited via Facebook advertisements. We proffer that trust plays a large role here; when participants were recruited either via AMT or snowball sampling (that is, had a mediating presence between us and the survey), they appeared much more willing to add the Facebook application and take part in the survey. There is potential for Facebook-based recruiting for cross-cultural studies, however further experimentation is required.

\section{References}

[1] R. Bickel, Multilevel analysis for applied research: It's just regression! ( $1^{\text {st }}$ ed.). New York: The Guilford Press, 2007.

[2] C. Liu, J.T. Marchewka, and C. Ku, American and Taiwanese perceptions concerning privacy, trust, and behavioral intentions in electronic commerce. Journal of Global Information Management, 12(1), p18-40, 2004. http://dx.doi.org/10.4018/jgim.2004010102.

[3] H. Cho, M. Rivera-Sánchez, and S.S. Lim, A multinational study on online privacy: Global concerns and local responses. New Media \& Society, $\quad 11(3), \quad$ p395-416, 2009. http:/dx.doi.org/10.1177/1461444808101618.

[4] H. Krasnova, and N.F. Veltri, Privacy calculus on social networking sites: Explorative evidence from Germany and USA. In Jr. Ralph H. Sprague (Ed.), Proceedings of the 2010 43rd Hawaii International Conference on System Sciences (HICSS) (p1-10). Hawaii, U.S.A.: IEEE Press, 2010. http://dx.doi.org/10.1109/HICSS.2010.307.

[5] P. Lowry, J. Cao, and A. Everard, Privacy concerns versus desire for interpersonal awareness in driving the use of self-disclosure technologies: The case of instant messaging in two cultures. Journal of Management Information Systems, 27(4), p163-200, 2011. http://dx.doi.org/10.2753/MIS0742-1222270406.

[6] Y.J. Park, Privacy regime, culture and user practices in the 
cyber-marketplace. info, $\quad 10(2), \quad$ p57-74, 2008. http://dx.doi.org/10.1108/14636690810862811.

[7] G.H. Hofstede, Culture's consequences: Comparing values, behaviors, institutions, and organizations across nations. Thousand Oaks, Calif.: Sage Publications, 2001.

[8] R. Thomson, and N. Ito, Socio-ecological structure and privacy concern on Facebook: The role of relational mobility. Presented at Cyberspace 2012, Brno, Czech Republic, November 30-December 2, 2012.

[9] R. Thomson, A socio-ecological approach to privacy concern on Facebook: The role of relational mobility. Masters Thesis, Hokkaido University, 2013.

[10] R.E. Wilson, S.D. Gosling, and L.T. Graham, A review of Facebook research in the social sciences. Perspectives on Psychological Science, 7(3), p203-220, 2012. http://dx.doi.org/10.1177/1745691612442904.

[11] M. Zuckerberg, One Billion People on Facebook. Retrieved on October 25 , 2012 , from http://newsroom.fb.com/news/2012/10/one-billion-people-on-facebook I.

[12] J. Kiss, Facebook UK loses 600,000 users in December. The Guardian. Retrieved on May 14, 2013, from http://www.guardian.co.uk/technology/2013/jan/14/facebook-loses-ukusers-december.

[13] S. Kitayama, Culture and basic psychological processes-toward a system view of culture: Comment on Oyserman et al. Psychological Bulletin, 128(1), p89-96, 2002. http://dx.doi.org/10.1037/0033-2909.128.1.89.

[14] M. Gjoka, M. Kurant, C.T. Butts, and A. Markopoulou, Practical recommendations on crawling online social networks. IEEE Journal on Selected Areas in Communications, 29(9), p1872-1892, 2011. http://dx.doi.org/10.1109/JSAC.2011.111011.

[15] Facebook, Facebook terms. Palo Alto, CA: Facebook. Retrieved on May 14, 2013, from https://www.facebook.com/legal/terms.

[16] S. Wolfram, Data science of the Facebook world. Stephen Wolfram Blog. Retrieved on May 14, 2013, from http://blog.stephenwolfram.com/2013/04/data-science-of-the-facebookworld/.

[17] A. Nazir, A. Waagen, V.S. Vijayaraghavan, C.-N. Chuah, R.M. D'Souza, and B. Krishnamurthy, Beyond friendship: Modeling user activity graphs on social network-based gifting applications. In J. Byers and J. Kurose (Eds.), Proceedings of the 2012 ACM conference on Internet measurement conference (p467-480). New York, NY, USA: ACM Press, 2012. http://dx.doi.org/10.1145/2398776.2398826. 
[18] A. Nazir, S. Raza, and C.-N. Chuah, Unveiling Facebook: a measurement study of social network based applications. In Tatul Mahajan and Alex C. Snoeren (Eds.), Proceedings of the 8th ACM SIGCOMM conference on Internet measurement (p43-56). New York, NY, USA: ACM 2008. http://dx.doi.org/10.1145/1452520.1452527.

[19] D. Stillwell, and M. Kosinski, M., Welcome to the myPersonality Project Website. Retrieved on May 8, 2013, from http://mypersonality.org/wiki/doku.php.

[20] D. Quercia, R. Lambiotte, D. Stillwell, M. Kosinski, and J. Crowcroft, The personality of popular Facebook users. In S. Poltrock and C. Simone (Ed.), Proceedings of the ACM 2012 conference on Computer Supported Cooperative Work (p955-964). New York, NY, USA: ACM Press, 2012. http://dx.doi.org/10.1145/2145204.2145346.

[21] H. Tan, H. Forgasz, G. Leder, and A. McLeod, Survey recruitment using Facebook: Three studies. Presented at the International Conference on Internet Studies, August 17-18, 2012, Bangkok.

[22] D.E. Ramo, and J.J. Prochaska, Broad reach and targeted recruitment using Facebook for an online survey of young adult substance use. Journal of Medical Internet Research, 14(1), 2012. http://dx.doi.org/10.2196/jmir.1878.

[23] L. Jones, B.I. Saksvig, M. Grieser, and D.R. Young, Recruiting adolescent girls into a follow-up study: Benefits of using a social networking website. Contemporary Clinical Trials, 33(2), p268-272, 2012. http://dx.doi.org/10.1016/j.cct.2011.10.011.

[24] Y. Fenner, S.M. Garland, E.E. Moore, Y. Jayasinghe, A. Fletcher, S.N. Tabrizi, and J. D. Wark, Web-based recruiting for health research using a social networking site: An exploratory study. Journal of Medical Internet Research, 14(1), 2012. http://dx.doi.org/10.2196/jmir.1978.

[25] J.M. Kapp, C. Peters, and D. P. Oliver, Research recruitment using Facebook advertising: Big potential, big challenges. Journal of Cancer Education, 28(1), p134-137, 2013. http://dx.doi.org/10.1007/s13187-012-0443-z.

[26] M. Kito, Shared and unique prototype features of relationship concepts and their roles in romantic relationship functioning. Doctoral dissertation, University of Manitoba, 2010.

[27] Facebook, Improve \& understand your ad or sponsored story's delivery. Retrieved on May 10, 2013, from https://www.facebook.com/help/415882831787433/.

[28] M. Buhrmester, T. Kwang, and S. D. Gosling, Amazon's mechanical Turk a new source of inexpensive, yet high-quality, data? Perspectives on Psychological Science, 6(1), p3-5, 2011. http://dx.doi.org/10.1177/1745691610393980. 
[29] W. Mason, and S. Suri, Conducting behavioral research on Amazon's Mechanical Turk. Behavior Research Methods, 44(1), p1-23, 2012. http://dx.doi.org/10.3758/s13428-011-0124-6.

[30] S.H. Batool, and K. Mahmood, Entertainment, communication or academic use? A survey of Internet café users in Lahore, Pakistan. Information Development, 26(2), p141-147, 2010. http://dx.doi.org/10.1177/0266666910366650.

[31] F. Shafique, and K Mahmood, Why the offline are offline: A survey of Internet use by Educated Pakistani Adults. CyberPsychology \& Behavior, 12(3), http://dx.doi.org/10.1089/cpb.2008.0122.

[32] ITU, Statistics. Retrieved on October 2, 2013, from http://www.itu.int/en/ITU-D/Statistics/Pages/stat/default.aspx.

[33] Social Bakers, Facebook Statistics and Metrics by Country. Retrieved on October 2, 2013, from http://www.socialbakers.com/facebook-statistics/.

[34] World Values Survey Association, World values survey 1981-2008 official aggregate v.20090901. ASEP/JDS, Madrid. Retrieved on October 2, 2013, from http://www.wvsevsdb.com/wvs/WVSData.jsp.

[35] UNDP, Gender inequality index (GII). Webpage. Retrieved on October 2, 2013, from http://hdr.undp.org/en/statistics/gii/. 
